\title{
Kolmogorov complexity and symmetric relational structures
}

\author{
W.L. Fouché \& P.H. Potgieter \\ Department of Quantitative Management, University of South Africa \\ PO Box 392, Unisarand 0003, Pretoria, South Africa \\ e-mail: $\{$ fouchwl,potgiph\}@unisa.ac.za
}

November 11, 2018

\begin{abstract}
We study partitions of Fraïssé limits of classes of finite relational structures where the partitions are encoded by infinite binary strings which are random in the sense of KolmogorovChaitin.
\end{abstract}

\section{Introduction}

This paper follows on [5] where a study was made of the properties of combinatorial configurations which are encoded or generated by infinite binary strings which are random in the sense of Kolmogorov-Chaitin 14, 2 (to be referred to as KC-strings ). We shall study countable homogeneous structures from this point of view. A relational structure $X$ is homogeneous if any isomorphism $f: A \rightarrow B$ between finite substructures of $X$ can be extended to an automorphism of $X$. This is perhaps the strongest symmetry condition one can impose on a relational structure. Our aim is to depict various situations where this kind of symmetry will be seen to be preserved by an arbitrary KC-string. Our work is based on Fraïssé's well-known characterisation of countable homogeneous structures [7.

A well-known example of a countable homogeneous structure is the random graph $R$ of Rado [17. We now illustrate some of the results of this paper with respect to the graph $R$. For a finite graph $\beta$, write $[R, \beta]$ for the set of copies of $\beta$ in $R$. We call a subset $Y$ of $[R, \beta]$ a $\beta$ organisation when $Y$ is exactly the set of all copies of $\beta$ in some subgraph $R^{\prime}$ of $R$, where $R^{\prime}$ is isomorphic to $R$. Now, $R$ has a simple recursive representation of the form $(\omega, E)$ where $E$ is a recursive subset of the set of 2 -subsets of $\omega$. This implies that one can find a recursive enumeration $\left(\beta_{j} \mid j<\omega\right)$, without repetition, of the set $[R, \beta]$. Let $\varepsilon=\prod_{j=0}^{\infty} \varepsilon_{j}$ be a KC-string. If we define a 2-colouring $\chi_{\varepsilon}:[R, \beta] \rightarrow\{0,1\}$ by giving each $\beta_{j}$ the colour $\varepsilon_{j}$, it will be shown that there always exists a monochromatic $\beta$-organisation $Y_{\varepsilon}$. Moreover, one can compute the $\beta$-organisation $Y_{\varepsilon}$ from $\varepsilon$ by means of a simple greedy algorithm. In this way, a KC-string has two aspects: (i) as a random partition of the copies of $\beta$ in $R$, and (ii) as a generator of a $\beta$-organisation in $R$ which is monochromatic under this partition. The symmetric structure $R$ is reflected (or preserved) by each KC-string in two distinct ways.

Similar results will be established for many other homogeneous structures. The main result is formulated in Section 2 and proved in Section 3. In Section 4 we apply this theory to the Fraïssé limits of what we shall call ranked diagrams. It is also shown how a KC-string can be used to generate the Fraïssé limit in this case. 


\section{Preliminaries}

The composition of two functions $f$ and $g$, denoted by $f g$, is defined by $f g(x)=f(g(x))$. The set of non-negative integers is denoted by $\omega$. We view the elements of $\omega$ as finite ordinals, so that $n<\omega$ denotes the set $\{0,1, \ldots, n-1\}$. The cardinality of a finite set $A$ is denoted by $|A|$. We write $\mathcal{N}$ for the product space $\{0,1\}^{\omega}$. The set of words over the alphabet $\{0,1\}$ is denoted by $\{0,1\}^{*}$. If $\alpha=\alpha_{0} \alpha_{1} \alpha_{2} \ldots$ is in $\mathcal{N}$ and $n<\omega$, we write $\bar{\alpha}(n)$ for the binary word $\prod_{j<n} \alpha_{j}$. We use the usual recursion-theoretic terminology $\Sigma_{r}^{0}, \Pi_{r}^{0}$ and $\Delta_{r}^{0}$ for the description of the arithmetical subsets of $\omega^{k} \times \mathcal{N}^{\ell}-$ see [10], for example. We write $\lambda$ for the Lebesgue measure on $\mathcal{N}$. This is the unique probability measure that assigns the value $\frac{1}{2}$ to each of the events $A_{i}=\left\{\alpha \in \mathcal{N} \mid \alpha_{i}=1\right\}$ and under which the events $A_{i}$ are statistically independent.

A prefix algorithm is a partial recursive function $f$ from $\{0,1\}^{*}$ to $\{0,1\}^{*}$ whose domain is prefix-free, i.e. if $u, v \in \operatorname{dom} f$ then neither is an initial segment of the other. It is well-known (and easy to prove) that there is an effective enumeration of prefix algorithms and, therefore, that there is some universal prefix algorithm $U$. For $s \in\{0,1\}^{*}$ let $H(s)$, the Kolmogorov-complexity of $s$, be the length of a shortest "program" $p \in\{0,1\}^{*}$, such that $U(p)=s$. (For the history and underlying intuition of these notions, the reader is referred to [20. See also [15], 2], 9] or [8].) An infinite binary string $\varepsilon$ is said to be Kolmogorov-Chaitin complex (KC-complex) if and only if

$$
\exists m \forall n H(\bar{\varepsilon}(n)) \geq n-m .
$$

The set of KC-complex strings does not depend on the choice of the universal prefix algorithm $U$ and has $\lambda$-measure one. We denote this set by $K C$ and refer to the elements as KC-strings. We shall make frequent use of the following result.

Theorem 1. [6] If $X$ is a $\Pi_{2}^{0}$-subset of $\mathcal{N}$ and $\lambda(X)=1$, then $X$ contains every $K C$-string $\varepsilon$.

The proof of this result is based on Martin-Löf's description [16] of the set $K C$.

In the sequel, $\mathcal{L}$ will stand for the signature of a relational structure. Moreover, $\mathcal{L}$ will always be finite and the arities of the relational symbols will all be $\geq 1$. This has the implication that the empty set carries a unique $\mathcal{L}$-structure. The definitions that follow were introduced by Fraïssé [7] in 1954. (For a general discussion of the results to be summarised, the reader is also referred to Hodges [1], Chapter 7 ).

The age of an $\mathcal{L}$-structure $X$, written $\operatorname{Age}(X)$, is the class of all finite $\mathcal{L}$-structures (defined on finite ordinals ) which can be embedded as $\mathcal{L}$-structures into $X$. The structure $X$ is homogeneous (some authors say ultrahomogeneous) if, given any isomorphism $f: A \rightarrow B$ between finite substructures of $X$, there is an automorphism $g$ of $X$ whose restriction to $A$ is $f$. The following result is due to Fraïssé. ( See 11], Chapter 7, for a proof. )

Proposition 1. The countable $\mathcal{L}$-structure $X$ is homogeneous if and only if, for $A, B \in \operatorname{Age}(X)$ and embeddings $f: A \rightarrow B, h: A \rightarrow X$, there is an embedding $g: B \rightarrow X$ such that $h=g f$. It suffices to require this when $|B|=|A|+1$.

A class $\mathbf{K}$ of finite $\mathcal{L}$-structures has the amalgamation property if, for structures $A, B_{1}, B_{2}$ in $\mathbf{K}$ and embeddings $f_{i}: A \rightarrow B_{i}(i=1,2)$ there is a structure $C$ in $\mathbf{K}$ and there are embeddings $g_{i}: B_{i} \rightarrow C(i=1,2)$, such that $g_{1} f_{1}=g_{2} f_{2}$.

Suppose $\mathbf{K}$ is a countable class of finite $\mathcal{L}$-structures, the domains of which are finite ordinals such that

1. if $A$ is a finite $\mathcal{L}$-structure defined on some finite ordinal, if $B \in \mathbf{K}$ and if there is an embedding of $A$ into $B$, then $A \in \mathbf{K}$;

2. the class $\mathbf{K}$ has the amalgamation property. 
Then, Fraïssé showed that there is a countable homogeneous structure $X$ such that $\operatorname{Age}(X)=\mathbf{K}$. Moreover, $X$ is unique up to isomorphism. The unique $X$ is called the Fraïssé limit of $\mathbf{K}$. We also recall that, conversely, the age $\mathbf{K}$ of a countable homogeneous structure has properties (i) and (ii).

In our study of partitions of a homogeneous structure $X$ we shall require its age to be dense in $X$ in the following sense: If $A, B \in \operatorname{Age}(X)$ and $i: A \rightarrow B$ is an embedding, then there exist $C \in \operatorname{Age}(X)$ and embeddings $f_{1}, f_{2}: B \rightarrow C$ such that $f_{1} i=f_{2} i$ and $\operatorname{Im} f_{1} \cap \operatorname{Im} f_{2}=\operatorname{Im} f_{1} i=\operatorname{Im} f_{2} i$. The Fraïssé limit of finite graphs ( the random graph of Rado [17] ) and the Fraïssé limit of ranked diagrams (see Section 4) are examples of homogeneous structures with dense ages. For any $n$, a disjoint union of countably many copies of the finite complete graph $K_{n}$ is an example of a homogeneous structure whose age is not dense. (The complement of this structure does have a dense age. ) The following combinatorial lemma plays a central role in the proof of Theorem 2

Lemma 1. Suppose $X$ is a countable homogeneous structure with a dense age. If $U$, $V$ are disjoint subsets of $X$, then there is a sequence $\left(V_{i} \mid i<\omega\right)$ of pairwise disjoint subsets of $X$ such that $U \cap V_{i}=\emptyset$ and $U \cup V_{i}$ and $U \cup V$ inherit isomorphic $\mathcal{L}$-structures from $X$, for all $i<\omega$.

Proof. Set $V_{0}=V$ and suppose pairwise disjoint $V_{0}, \ldots, V_{k-1}$ have been constructed with $U \cap V_{i}=\emptyset$ and $U \cup V_{i}$ isomorphic to $U \cup V$ for all $i<k$. Set $W=\bigcup_{i<k} V_{i}$. Choose $A, B \in \operatorname{Age}(X)$ with $A \subset B$ so that $A$ is isomorphic to $U \subset X$ via an isomorphism which extends to an isomorphism of $B$ to $U \cup W \subset X$. Since Age $(X)$ is dense in $X$, there exist $C \in \operatorname{Age}(X)$ and embeddings $f_{1}, f_{2}: B \rightarrow C$ such that $A \subset C$ and $f_{1}, f_{2}$ are both the identity on $A$, while $\operatorname{Im} f_{1}$ and $\operatorname{Im} f_{2}$ will have exactly the elements of $A$ in common.

For $i \in\{1,2\}$, let $A_{i}$ be the complement of $A$ in $\operatorname{Im} f_{1}$. Then $A_{1} \cap A_{2}=\emptyset$ but $A \cup A_{i}$ is isomorphic to $B$ and hence also to $U \cup W \subset X$. Moreover, $A \cap A_{i}=\emptyset$. Let $\alpha$ be an isomorphism (e.g. the one from the construction of $A$ and $B$ above) from $B$ onto $U \cup W \subset X$ that maps $A$ onto $U$. By Proposition 1 there is an embedding $\beta$ such that the following diagram commutes.

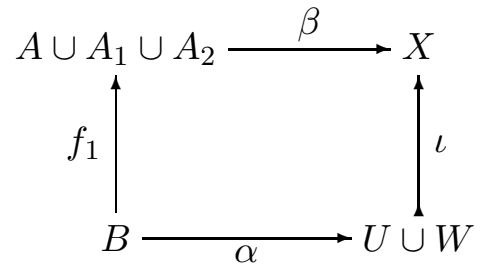

We can write $\operatorname{Im} \beta=U \cup W \cup W^{\prime}$ with $U \cup W^{\prime}$ isomorphic to $U \cup W$ and $(U \cup W) \cap W^{\prime}=\emptyset$. Let $V_{k}$ be any subset of $W^{\prime}$ such that $U \cup V_{k}$ is isomorphic to $U \cup V$. (Such as exists by the isomorphism of $U \cup W$ with $U \cup W^{\prime}$.) The sequence $\left(V_{i} \mid i<\omega\right)$ constructed in this way has the required properties.

A recursive representation of a countable $\mathcal{L}$-structure $X$ is a bijection $\phi: \mathrm{X} \rightarrow \omega$ such that, for each $R \in \mathcal{L}$, if the arity of $R$ is $n$, then the relation $R^{\phi}$ defined on $\omega^{n}$ by

$$
R^{\phi}\left(x_{1}, x_{2}, \ldots, x_{n}\right) \leftrightarrow R\left(\phi^{-1}\left(x_{1}\right), \ldots, \phi^{-1}\left(x_{n}\right)\right)
$$

is recursive. If we identify the underlying set of $X$ with $\omega$ via $\phi$ and each $R$ with $R^{\phi}$ we call the resulting structure a recursive $\mathcal{L}$-structure.

If $X$ is countable and homogeneous and if $\operatorname{Age}(X)$ has an enumeration $A_{0}, A_{1}, A_{2}, \ldots$, possibly with repetition, with the property that there is a recursive procedure that yields, for each $i<\omega$, and $R \in \mathcal{L}$, the underlying set $n(i)$ of $A_{i}$ together with the interpretation of $R$ in $n(i)$, then we call $\left(A_{i} \mid i<\omega\right)$ a recursive enumeration of $\operatorname{Age}(X)$. It follows from the construction of Fraïssé limits from their ages, as discussed in [1] (p329) that one can construct a recursive representation of $X$ from a recursive enumeration of its ages. (Conversely, it is trivial to derive a recursive enumeration 
of $\operatorname{Age}(X)$ from a recursive representation of $X$.) It is therefore not difficult to find recursive representations for Fraïssé limits of classes $\mathbf{K}$ from recursive enumerations of their members.

Let $X$ be a countable, homogeneous structure with a recursive representation $\phi$. For $\beta \in$ Age $(X)$, let $[X, \beta]$ be the set of copies (images under embeddings) of $\beta$ in $X$. Suppose, in addition, that $X$ has a dense age. We can use $\phi$ to find a recursive enumeration $\beta_{0}, \beta_{1}, \ldots$, without repetition, of the set $[X, \beta]$. The density of $\operatorname{Age}(X)$ in $X$ ensures that $[X, \beta]$ is infinite (see Lemma 1) and the representation $\phi$ can be used to decide whether a given finite subset of $X$ inherits a structure isomorphic to $\beta$.

If $\alpha$ is an infinite binary string then $\alpha$ induces a 2-colouring $\chi_{\alpha}$ of $[X, \beta]$ where $\chi_{\alpha}$ assigns to the $i$-th copy $\beta_{i}$ of $\beta$ in $X$ the colour $\alpha_{i}$, the $i$-th bit of $\alpha$. The main theorem of the paper can now be formulated.

Theorem 2. Let $X$ be a recursive homogeneous structure with a dense age. For each $\beta \in \operatorname{Age}(X)$ and each $K C$-string $\varepsilon$, there exists an embedding $\nu: X \rightarrow X$ such that $\chi_{\varepsilon}\left(\beta^{\prime}\right)=1$ for each $\beta^{\prime} \in[\nu(X), \beta]$. In addition, $\nu$ can be so constructed that it is recursive relative to $\varepsilon$.

One can think of the mappings $\chi_{\alpha}:[X, \beta] \rightarrow 2$ as random partitions. It follows from Theorem 2 that when $[X, \beta]$ is subjected to a random partitioning then, with probability 1 , one can find copies $X_{0}, X_{1}$ of $X$ in $X$ such that $\chi_{\alpha}$ is of colour $i$ on $\left[X_{i}, \beta\right](i=0,1)$. This is because $\alpha$ is in $K C$ with probability 1 . Moreover, when $\alpha$ is a KC-string, we can effectively generate, relative to $\alpha$, the automorphic copies $X_{0}$ and $X_{1}$ of $X$. The proof of the theorem appears in Section 3 ,

Recall that Ramsey's Theorem [18] says that for $X=K_{\omega}$, the complete graph on the natural numbers, for $\beta=K_{n}$, the complete graph on $n$ points, and $\varepsilon$ an arbitrary binary sequence, there exists an embedding $\nu: X \rightarrow X$ such that $[\nu(X), \beta]$ is monochromatic under the 2-colouring of $[X, \beta]$ induced by $\varepsilon$. E. Specker [19] has observed that there exists a recursive sequence $\varepsilon$ such that, for the colouring of $\left[X, K_{2}\right]$ induced by $\varepsilon$, there exists no recursive copy $X^{\prime}$ of $X$ such that $\left[X^{\prime}, K_{2}\right]$ is monochromatic. This has been further refined by C.G. Jockusch [12] who showed that there exists a recursive sequence $\varepsilon$ such that, for the colouring of $\left[X, K_{n}\right]$ induced by $\varepsilon$, there is no $\Sigma_{n}^{0}$ copy $X^{\prime}$ of $X$ for which $\left[X^{\prime}, \beta\right]$ is monochromatic. However, for any recursive $\varepsilon$, there always exists a $\Pi_{n}^{0}$ copy $X^{\prime}$ of $X$ for which $\left[X^{\prime}, \beta\right]$ is monochromatic. It follows, however, from Theorem 2 that when $\varepsilon$ is a KC-string, one can find a monochromatic $X^{\prime}$ which is recursive in $\varepsilon$. This emphasises that Jockusch's results exploit the non-random nature of recursive partitions.

\section{Complex partitions of Fraïssé limits}

In the following we will denote the class of all finite subsets of a set $Y$ by Fin $Y$. If $w \in$ Fin $\omega$ we denote the largest element of $w$ by $\max w$. If $w$ is empty, then $\max w=-1$. If $n \in \omega$, then by $w n$ we mean $w \cup\{n\}$. We write $v<w$ if there is a $t \neq \emptyset$ with $w=v \cup t$ and $\max v<\min t$.

Definition 1. Let $Y$ be a countably infinite set. An encoding of $Y$ is a function $\pi:$ Fin $\omega \rightarrow$ Fin $Y$ such that

(i) $\pi(\emptyset)=\emptyset$ and for some $w_{0} \in$ Fin $\omega$,

$$
\pi\left(w_{0}\right) \neq \emptyset
$$

(ii) whenever $n>m>\max w$

$$
\pi(w n) \cap \pi(w m)=\pi(w)
$$

(iii) for each $w$ with $\pi(w) \neq \emptyset$,

$$
\sum 2^{-|\pi(w k) \backslash \pi(w)|}=\infty
$$

where the summation is over all $k>\max w$ such that $\pi(w k) \neq \pi(w)$. 
Definition 2. An encoding $\pi$ is called effective relative to a bijection

$\sigma: \omega \rightarrow Y$ when there exist a recursive binary relation $R_{\sigma}$ and a recursive function $f$, such that, for $i \in \omega$ and $w \in$ Fin $\omega$,

(i) $R_{\sigma}(i, w) \leftrightarrow \sigma(i) \in \pi(w)$,

and also

(ii) $f(w)=|\pi(w)|$.

These definitions have been adapted from [5]. The next theorem is a generalization of Theorem A of [5].

Theorem 3. If the encoding $\pi:$ Fin $\omega \rightarrow$ Fin $Y$ is effective relative to $\sigma$ and if $\varepsilon \in K C$, then there exists a strictly increasing sequence

$$
w_{1}<w_{2}<w_{3}<\ldots
$$

in Fin $\omega$ such that

$$
\varepsilon(j)=1 \text { whenever } \sigma(j) \in \bigcup_{n \geq 1} \pi\left(w_{n}\right) .
$$

There exists an oracle computation of this sequence from $\varepsilon$.

Proof. Let $\pi$ be an encoding which is effective relative to $\sigma$, as defined above. Apply (11) to fix $w_{0}=v_{0} k \in$ Fin $\omega$, where $k=\max w_{0}$, such that $\pi\left(v_{0}\right)=\emptyset$ but $\pi\left(v_{0} k\right) \neq \emptyset$.

Let $\varepsilon$ be in $K C$. We construct a strictly increasing sequence in Fin $\omega$ by induction so that for each $n$

$$
w_{0}<w_{1}<\ldots<w_{n} \text { and } \varepsilon(j)=1 \text { for all } \sigma(j) \in \bigcup_{k=1}^{n} \pi\left(w_{k}\right) .
$$

The construction will be recursive in $\varepsilon$. This will suffice to prove the theorem.

Suppose $n \geq 0$ and $w_{0}, \ldots, w_{n}$ have been constructed. For every $k>\max w_{n}$, we define $B_{k} \subseteq \mathcal{N}$ by:

$$
\alpha \in B_{k} \leftrightarrow(\forall j)\left[\sigma(j) \in \pi\left(w_{n} k\right) \backslash \pi\left(w_{n}\right) \rightarrow \alpha_{j}=1\right] .
$$

By Definition 2 $R_{\sigma}(i, w)$ and the function $w \mapsto|\pi(w)|$ are both recursive, so there exists a total recursive function $\psi: \omega \rightarrow \omega$ such that $j \leq \psi(k)$ whenever $\sigma(j) \in \pi\left(w_{n} k\right)$. The function $\psi$ could, for example, compute the largest $j$ so that $\sigma(j) \in \pi\left(w_{n} k\right)$ when $w_{n}$ and $k$ have been given. Now,

$$
\alpha \in B_{k} \leftrightarrow(\forall j \leq \psi(k))\left[R_{\sigma}\left(j, w_{n} k\right) \wedge \neg R_{\sigma}\left(j, w_{n}\right) \rightarrow \alpha_{j}=1\right] .
$$

It now follows that the relation $\alpha \in B_{k}$ is recursive in $k$ and $\alpha$.

We shall define a sequence $X_{0}, X_{1}, X_{2}, \ldots$ of statistically independent random variables on the probability space $(\mathcal{N}, \Sigma, \lambda)$ where $\Sigma$ is the collection of Borel subsets of $\mathcal{N}$ and $\lambda$ the Lebesgue measure, as before. Let $X_{i}(\alpha)=\alpha_{i}$ for $\alpha \in \mathcal{N}$ and $i \in \omega$. If $k>\ell>\max w_{n}$ and both $\pi\left(w_{n} k\right) \neq \pi\left(w_{n}\right)$ and $\pi\left(w_{n} \ell\right) \neq \pi\left(w_{n}\right)$, then the events $B_{k}$ and $B_{\ell}$ are statistically independent. To see this, note that $B_{k}$ belongs to the $\sigma$-algebra generated by

$$
\left\{X_{j} \mid \sigma(j) \in \pi\left(w_{n} k\right) \backslash \pi\left(w_{n}\right)\right\}
$$


and $B_{\ell}$ belongs to the $\sigma$-algebra generated by

$$
\left\{X_{j} \mid \sigma(j) \in \pi\left(w_{n} \ell\right) \backslash \pi\left(w_{n}\right)\right\} .
$$

Independence follows from the fact that $\pi\left(w_{n} k\right) \cap \pi\left(w_{n} \ell\right)=\pi\left(w_{n}\right)$.

Since the probability

$$
P\left(\alpha \in B_{k}\right)=2^{-\left|\pi\left(w_{n} k\right) \backslash \pi\left(w_{n}\right)\right|}
$$

and we know, by (3), that the sum of the probabilities of these independent events diverges, it follows from the second Borel-Cantelli lemma that the event $B_{k}$, with $\pi\left(w_{n} k\right) \neq \pi\left(w_{n}\right)$, occurs infinitely often with probability 1 . In particular, if we define $B$ by

$$
\alpha \in B \leftrightarrow \exists k\left(k>\max w_{n} \wedge \pi\left(w_{n} k\right) \neq \pi\left(w_{n}\right) \wedge \alpha \in B_{k}\right)
$$

then $\lambda(B)=1$. But $B$ is a $\Sigma_{1}^{0}$-set and $\Sigma_{1}^{0} \subset \Pi_{2}^{0}$, so it follows directly from Theorem 1 that $\varepsilon \in B$. Choose the smallest $k>\max w_{n}$ for which $\pi\left(w_{n} k\right) \neq \pi\left(w_{n}\right)$ and such that $\varepsilon \in B_{k}$. Set $w_{n+1}=w_{n} k$. Then $\varepsilon(j)=1$ for all $j$ with $\sigma(j) \in \cup_{\ell \leq n+1} \pi\left(w_{\ell}\right)$. Every step - including this last one - is effective relative to $\varepsilon$.

We now proceed to prove the main theorem of the paper (Theorem 2).

Proof. Let $X$ be a recursive homogeneous structure with a dense age. There is a universal procedure that yields, for finite subsets $U, V$ of $X$ with $U \cap V=\emptyset$ and each $k<\omega$ a set $V_{k}$ such that the sequence $\left(V_{k} \mid k<\omega\right)$ is as in the conclusion of Lemma 1. This is evident from the proof of Lemma 1] since the inductive constructions of the $V_{k}$ can be done recursively for a given recursive structure $X$.

Since $X$ is recursive we can identify its domain with $\omega$. Our aim is to construct a function $\mu$ : Fin $\omega \rightarrow$ Fin $\omega$ such that, for $w \in$ Fin $\omega$, there is an embedding $\nu(\omega)$ from the $\mathcal{L}$-structure on $|w| \subset X$ to an $\mathcal{L}$-structure $\mu(w) \subset X$ such that, for $k>\max w$, the embedding $\nu(w k)$ will be an extension of $\nu(w)$.

The construction will be such that if $n>m>\max w$ then

$$
\mu(w n) \cap \mu(w m)=\mu(w)
$$

and $\mu(w m)$ will always contain a copy of $\beta$ which is not in $\mu(w)$. Finally, we shall ensure that that the embeddings $\nu(w)$ will depend recursively on $w$. The construction is as follows:

(1) Set $\mu(\emptyset)=\emptyset$ and $\nu(\emptyset)=\emptyset$.

(2) Assume $\mu(w), \nu(w)$ and $k>\max w$ are given. Construct $V$ (which will be a finite set) such that $V \cap \mu(w)=\emptyset$ and if we set $Z=\mu(w) \cup V$ then $Z$ contains a copy of $|w|+1$, extending the copy of $|w|$ in $\mu(w)$, and $Z$ contains a copy of $\beta$ not in $\mu(w)$. (Proposition 1] shows that we can extend $|w|$ and Lemma 1 implies that there are infinitely many copies of $\beta$.) Next, construct a pairwise disjoint sequence $V_{0}, V_{1}, V_{2}, \ldots$ (again using Lemma 1) which are all also disjoint from $\mu(w)$, such that if we set $Z_{j}=\mu(w) \cup V_{j}$ then $Z_{j}$ is isomorphic to $Z$. Finally, set $\mu(w k)=Z_{k}$ and let $\nu(w k)$ be an embedding of $|w|+1$ into $Z_{k}$ which extends $\nu(w)$.

Set $\pi(w)=[\mu(w), \beta]$. We now show that $\pi$ is an encoding of $Y=[X, \beta]$ in the sense of Definition 11. By the construction we see immediately that $\pi$ satisfies conditions (11) and (2) of Definition (1) In order to verify the condition (3), we note that if $n>\max w$ then $\pi(w n) \backslash \pi(w)$ is non-empty 
and its size is independent of $n$ (again by Step 2 of the construction). The divergence of the series follows.

Let $\beta_{0}, \beta_{1}, \ldots$ be an effective enumeration without repetition of $Y$. For $i<\omega$, set $\sigma(i)=\beta_{i}$. Note that, since we have an effective representation of $X$, the straight-forward (greedy!) algorithm for giving $\mu$ and $\pi$, respectively, shows that both are recursive. Since $\pi$ is recursive, so is the mapping $w \mapsto|\pi(w)|$. Also, whether $[\sigma(i) \in \pi(w)]$ holds, can be determined by listing and comparing the elements of $\sigma(i)$ and $\mu(w)$, where $\mu$ is as above. Therefore, $\pi$, as defined, is effective relative to $\sigma$ (in the sense of Definition [2).

Theorem 3 now gives an oracle computation of a strictly increasing sequence $w_{1}<w_{2}<w_{3}<\ldots$ from $\varepsilon$ such that $\varepsilon(j)=1$ whenever $\sigma(j) \in \cup \pi\left(w_{n}\right)$. In other words, since $\mu\left(w_{n}\right)$ is increasing in $n$, if $\sigma(j) \subset \cup \mu\left(w_{n}\right)$ then $\varepsilon(j)=1$.

The embeddings $\nu\left(w_{n}\right):\left|w_{n}\right| \rightarrow \mu\left(w_{n}\right)$ are mutually compatible and thus define an embedding $\nu: X \rightarrow X$ such that $\operatorname{Im} \nu \subset \bigcup_{n} \mu\left(w_{n}\right)$. This embedding $\nu$ is the required embedding, which is indeed recursive relative to $\varepsilon$ since $w \mapsto \nu(w)$ is recursive and the sequence $w_{1}<w_{2}<w_{3}<\ldots$ is recursive relative to $\varepsilon$.

\section{Ranked diagrams}

In [5] it was shown that partitioning the edges of the complete countable graph $K_{\omega}$ into two classes $E_{0}, E_{1}$ by means of a KC-string $\varepsilon$ yields two graphs $\left(\omega, E_{0}\right)$ and $\left(\omega, E_{1}\right)$ both of which are isomorphic to the Fraïssé limit of finite graphs. In this section we want to do the same for so-called ranked diagrams. These structures can be viewed as the Hasse diagrams of posets.

\subsection{An $\aleph_{0}$-categorical first-order theory of ranked diagrams.}

In the sequel, $\ell \geq 2$ is fixed. Let $\mathcal{L}$ be the signature having $\ell$ unary relations, $L_{0} \ldots L_{\ell-1}$ (denoting the levels of the ranked diagram), and one binary relation, $S$ (succession). The theory, $R D_{\ell}$, of ranked diagrams on $\ell$ levels ( $\ell$-diagrams), has the following three axioms :

(i) For all $x: L_{0}(x) \vee \ldots \vee L_{\ell-1}(x)$

(ii) For all $x$ :

$$
\bigwedge_{0 \leq i<j<\ell} \neg\left[L_{i}(x) \wedge L_{j}(x)\right]
$$

(iii) For all $x$ and $y$ :

$$
S(x, y) \rightarrow \bigwedge_{i=0}^{\ell-2}\left[L_{i}(x) \rightarrow L_{i+1}(y)\right]
$$

The preceding axioms imply that there exists, for each $x$, a unique $L_{i}$ such that $L_{i}(x)$ holds ( or - in different notation $-x \in L_{i}$ ) and also that $S(x, y)$ can hold only if $x$ and $y$ are on adjacent levels, $y$ being "above" $x$. A model of the theory $R D_{\ell}$ is an $\ell$-diagram. (A special class of these diagrams, namely the $k$-layered posets, has been investigated in 11.)

We shall identify a class of countable $\ell$-diagrams, having the property that each one of them also contains a copy of every other countable $\ell$-diagram. This class is defined by an $\aleph_{0}$-categorical first-order theory consisting of the axioms of $R D_{\ell}$ as well as a collection of extension axioms similar to the extension axioms used by Compton [4] in his proof of the fact that the class of partial orders has a (labelled) first order 0-1 law. In view of the result of Kleitman and Rothschild 


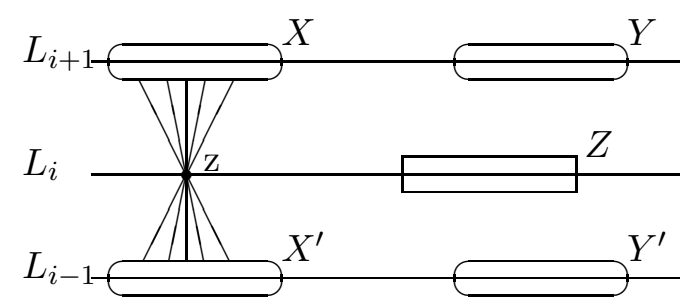

Figure 1: The extension axioms assert the existence of such a $z$ for any $X, Y, X^{\prime}, Y^{\prime}, Z$.

13], showing that a finite partial order will be ranked and of height 3 with labelled asymptotic probability 1 , it makes sense to investigate random partial orders via $\ell$-diagrams.

We now single out those $\ell$-diagrams that are not only models of $R D_{\ell}$ but also satisfy the following countable collection of axioms (indexed by the cardinalities of $X, Y, X^{\prime}, Y^{\prime}, Z$, for example ):

(iv) (Extension Axioms) For each $i<\ell$ and configuration of non-negative integers, $\left(n_{1}, n_{2}, n_{3}, n_{4}, n_{5}\right)$, an axiom stating that when $X, Y$ are disjoint subsets of $L_{i+1}, Z$ is a subset of $L_{i}$ and $X^{\prime}, Y^{\prime}$ disjoint subsets of $L_{i-1}$ such that $\left(|X|,|Y|,|Z|,\left|X^{\prime}\right|,\left|Y^{\prime}\right|\right)=\left(n_{1}, n_{2}, n_{3}, n_{4}, n_{5}\right)$ then, for some $z \in L_{i}$ such that $z \notin Z$, we have

$$
\begin{aligned}
& S(z, x), \quad S\left(x^{\prime}, z\right), \quad \neg S(z, y) \quad \text { and } \neg S\left(y^{\prime}, z\right) \\
& \text { for all } x \in X, \quad x^{\prime} \in X^{\prime}, \quad y \in Y \quad \text { and } y^{\prime} \in Y^{\prime}
\end{aligned}
$$

respectively. (See Figure 1.) We think of $L_{i-1}$, respectively $L_{i+1}$, as a name for the empty set when $i=0$, respectively $i=\ell-1$.

These extension axioms guarantee that we can extend a given arbitrary finite configuration on levels $i-1, i, i+1$ in the required way ( to a new $\ell$-diagram ) by just finding an appropriate $z$ on level $i$. Axioms (i)-(iv) all together give a countable collection of first-order sentences in our language $\mathcal{L}$. These make up a theory $\mathrm{T}_{\ell}$. We shall call its countable models the generic $\ell$-diagrams. Instances of the form $X=X^{\prime}=Y=Y^{\prime}=\emptyset$ of (iv) guarantee that in any model of $\mathrm{T}_{\ell}$, the unary relations $L_{i}$ are modelled by infinite sets, so that any countably infinite model necessarily has infinitely many elements on each level.

\subsection{Explicit construction of an generic $\ell$-diagram.}

We now give an example of how to construct a recursive object that represents a generic $\ell$-diagram. A similar construction can be given for Rado's random graph [17]. Let $A=\ell \times \omega$ be our underlying set and fix a collection

$$
p(i, n) \quad i \in \ell, \quad n<\omega
$$

of distinct odd primes. Now define the binary relation $P_{\ell}$ on $\ell \times \omega$ by

$$
(i, n) P_{\ell}(i+1, m) \leftrightarrow\left(\begin{array}{c}
m \neq 0 \text { and } p(i, n) \mid m \\
\text { OR } \\
n \neq 0 \text { and } p(i+1, m) \mid n
\end{array}\right) .
$$

In order to verify that $\left(A, P_{\ell}\right)$ is generic, we need to check the extension property (iv). We first assume $0<i<\ell-1$. Take any finite subsets

$$
X=\left\{\left(i+1, x_{0}\right), \ldots,\left(i+1, x_{p}\right)\right\}
$$




$$
\begin{aligned}
Y & =\left\{\left(i+1, y_{0}\right), \ldots,\left(i+1, y_{q}\right)\right\} \\
Z & =\left\{\left(i, z_{0}\right), \ldots,\left(i, z_{r}\right)\right\} \\
X^{\prime} & =\left\{\left(i-1, x_{0}^{\prime}\right), \ldots,\left(i-1, x_{s}^{\prime}\right)\right\} \\
Y^{\prime} & =\left\{\left(i-1, y_{0}^{\prime}\right), \ldots,\left(i-1, y_{t}^{\prime}\right)\right\}
\end{aligned}
$$

of $\ell \times \omega$ such that $X \cap Y=\emptyset=X^{\prime} \cap Y^{\prime}$. It is sufficient to show that there exists $z \notin\left\{0, z_{0}, \ldots, z_{r}\right\}$ so that

$$
\begin{array}{l|ll}
p\left(i+1, x_{k}\right) \mid z & , k \leq p \\
p\left(i-1, x_{k}^{\prime}\right) \mid z & , k \leq s \\
p\left(i+1, y_{k}\right) & \backslash z & , k \leq q \\
p\left(i-1, y_{k}^{\prime}\right) & \backslash \chi & , k \leq t
\end{array}
$$

and also

$$
\begin{array}{llll}
p(i, z) \backslash y_{k} \text { or } & y_{k}=0 & , k \leq q \\
p(i, z) \backslash y_{k}^{\prime} \text { or } & y_{k}^{\prime}=0 & , k \leq t
\end{array}
$$

This can be achieved by setting

$$
z=\left(\prod_{k \leq p} p\left(i+1, x_{k}\right)\right) \cdot\left(\prod_{k \leq s} p\left(i-1, x_{k}^{\prime}\right)\right) \cdot 2^{w}
$$

where $w$ has been chosen sufficiently large to make $z \neq z_{0}, \ldots, z_{r}$ and for $p(i, z)$ not to divide any of the non-zero second components of elements of $Y \cup Y^{\prime}$. This determines a $z$ with the required properties. The cases $i=0$ and $i=\ell-1$ are similarly dealt with.

\subsection{Application of Theorem 2 to ranked diagrams.}

Let $X$ be a generic $\ell$-diagram. If $A$ is a finite $\ell$-diagram and $f: A \rightarrow X$ any embedding, and if $B$ is a $\ell$-diagram with $|B|=|A|+1$ and $B \supset A$, then it follows directly from the extension axioms (iv) that $f$ can be extended to an embedding of $B$ into $X$. Since each singleton $\ell$-diagram can be embedded into $X$, it thus follows upon induction that any finite $\ell$-diagram can be embedded into $X$. Finally, it follows from Proposition 1 that $X$ is homogeneous. We conclude that $X$ is the Fraïssé limit of finite $\ell$-diagrams. We note that $\operatorname{Age}(X)$ is dense in $X$ so that Theorem 2 also applies to generic $\ell$-diagrams.

\subsection{Binary sequences that generate generic $\ell$-diagrams.}

Fix some canonical recursive bijection

$$
\psi:(\ell-1) \times \omega \times \omega \rightarrow \omega .
$$

Given $\alpha \in \mathcal{N}$ we generate a ranked diagram $S_{\alpha}$ on the underlying set $A=\ell \times \omega$ by putting

$$
(i, n) S_{\alpha}(i+1, m) \quad \text { whenever } \quad \alpha_{\psi(i, n, m)}=1 .
$$


We would now like to know for which $\alpha \in \mathcal{N}$, the ranked diagram $\left(A, S_{\alpha}\right)$ generated by the binary sequence $\alpha$ is $\ell$-generic, where $A=\ell \times \omega$, as before. Let

$$
G=\left\{\alpha \in \mathcal{N} \mid\left\langle A, S_{\alpha},\{0\} \times \omega, \ldots,\{\ell-1\} \times \omega\right\rangle \text { is a model for } T_{\ell}\right\} .
$$

The construction of $S_{\alpha}$, as in equation (5), is already such that the axioms (i)-(iii) of $T_{\ell}$ are automatically satisfied for all $\alpha$.

Let $P\left(\alpha, X, Y, Z, X^{\prime}, Y^{\prime}, z\right)$ stand for the predicate over $\mathcal{N} \times(\text { Fin } A)^{5} \times A$ which states that $z \notin Z$ and

$$
S_{\alpha}(z, x), \quad S_{\alpha}\left(x^{\prime}, z\right), \quad \neg S_{\alpha}(z, y) \quad \text { and } \neg S_{\alpha}\left(y^{\prime}, z\right)
$$

holds, for all $x \in X, x^{\prime} \in X^{\prime}, y \in Y$ and $y^{\prime} \in Y^{\prime}$ respectively. If we identify Fin $A$ with $\omega$ via a recursive bijection, then it is clear that $P$ is a recursive predicate. Set $K_{i}=\{i\} \times \omega$ for $i<\ell$ and $K_{-1}=K_{\ell-1}=\emptyset$. Let $Q(\alpha)$ be the predicate

$$
\begin{gathered}
(\forall 0 \leq i<l)\left(\forall X \in \operatorname{Fin} K_{i+1}\right)\left(\forall Y \in \operatorname{Fin} K_{i+1}\right)\left(\forall Z \in \operatorname{Fin} K_{i}\right)\left(\forall X^{\prime} \in \operatorname{Fin} K_{i-1}\right) \\
\left(\forall Y^{\prime} \in \operatorname{Fin} K_{i-1}\right)\left(\exists z \in K_{i}\right)\left(X \cap Y=X^{\prime} \cap Y^{\prime}=\emptyset \rightarrow P\left(\alpha, X, Y, Z, X^{\prime}, Y^{\prime}, z\right)\right)
\end{gathered}
$$

which is to say that $Q(\alpha)$ holds if and only if $\alpha$ codes a generic $\ell$-diagram. It is clear that $Q$ is a $\Pi_{2}^{0}$-predicate. We have thus shown that

Lemma 2. $G$ is a $\Pi_{2}^{0}$-set.

Let us now consider the probability that a uniformly randomly generated $\alpha$ will give an $\ell$-generic $\mathrm{RD}$ on $A$, where our probability measure is the Lebesgue measure $\lambda$, as before.

Lemma 3. With probability 1 , a sequence $\alpha \in \mathcal{N}$ defines a generic $\ell$-diagram.

Proof. We have to show that $\lambda(G)=1$. Note that

$$
G=\bigcap \bigcup_{z \in K_{i}}\left\{\alpha \mid P\left(\alpha, X, Y, Z, X^{\prime}, Y^{\prime}, z\right)\right\}
$$

where the intersection runs over all $i, X, Y, Z, X^{\prime}, Y^{\prime}$ such that $0 \leq i<\ell ; X, Y \in$ Fin $K_{i+1}$; $Z \in$ Fin $K_{i} ; X^{\prime}, Y^{\prime} \in$ Fin $K_{i-1}$ such that $X \cap Y=X^{\prime} \cap Y^{\prime}=\emptyset$.

Since this is a countable intersection, we can henceforth regard all parameters, save $z$, as fixed, and need only prove that

$$
\lambda\left(\bigcup_{z \in K_{i} \backslash Z}\left\{\alpha \mid P\left(\alpha, X, Y, Z, X^{\prime}, Y^{\prime}, z\right)\right\}\right)=1
$$

when $X, Y, X^{\prime}, Y^{\prime}$ are as above.

Now, if $z^{\prime}$ and $z^{\prime \prime}$ are distinct elements of $K_{i} \backslash Z$, then $P\left(\alpha, X, Y, Z, X^{\prime}, Y^{\prime}, z^{\prime}\right)$ holding for $\alpha$ and $P\left(\alpha, X, Y, Z, X^{\prime}, Y^{\prime}, z^{\prime \prime}\right)$ holding for $\alpha$ are independent events. For, the evaluation of these two instances of the predicate reference disjoint (finite) sets of digits in the sequence $\alpha$ ( $\psi$ above being one-to-one ). In each case, the probability that $P$ holds is $2^{-n}$ where $n=|X|+|Y|+\left|X^{\prime}\right|+\left|Y^{\prime}\right|$. We may therefore apply the second Borel-Cantelli lemma to conclude that the union,

$$
\bigcup_{z \in K_{i} \backslash Z}\left\{\alpha \mid P\left(X, Y, Z, X^{\prime}, Y^{\prime}, z\right)\right.
$$

does indeed have measure 1 , which proves the lemma.

Theorem 1 together with Lemmas 2 and 3 now immediately give the following theorem.

Theorem 4. If $\alpha$ is a $K C$-string, then the ranked diagram $\left(A, S_{\alpha}\right)$ is $\ell$-generic. 


\section{References}

[1] G. Brightwell H.J. Prömel A. Steger, 'The Average Number of Linear Extensions of a Partial Order', J. Comb. Th. Series A 73 (1996) 193-206.

[2] G.J. Chaitin, Algorithmic Information Theory (Cambridge University Press, 1987).

[3] C.C. Chang H.J. Keisler, Model Theory (North Holland, Amsterdam, 1973).

[4] K.J. Compton, 'Laws in Logic and Combinatorics', in I. Rival (ed) , Algorithms and Order (Kluwer Acad. Publ., Dordrecht, 1989) 353-383.

[5] W.L. Fouché, 'Descriptive Complexity and Reflective Properties of Combinatorial Configurations', Journal of the London Mathematical Society (2) 54 (1996) 199-208.

[6] W.L. Fouché, 'Identifying randomness given by high descriptive complexity', Acta Applicandae Mathematicae 34 (1994) 313-328.

[7] R. Fraïssé, 'Sur l'extension aux relations de quelques propriétés des ordres', Ann. Sci. École Norm. Sup. 71 (1954) 363-388.

[8] P. Gács, 'Randomness and Probability - Complexity of Description', Encyclopedia of Statistical Sciences (John Wiley \& Sons, 1986) 551-555.

[9] P. Gács, A review of G. Chaitin's Algorithmic Information Theory, Journal of Symbolic Logic 54 (1989) 624-627.

[10] P.G. Hinman, Recursion-theoretic Hierarchies (Springer, New York, 1978).

[11] W. Hodges, Model Theory, (Cambridge University Press, Cambridge, 1993).

[12] C.G. Jockusch jr., 'Ramsey's Theorem and Recursion Theory', Journal of Symbolic Logic 37 (1972) 268-280.

[13] D.J. Kleitman B.L. Rothschild, 'Asymptotic Enumeration of Partial orders on a Finite Set', Trans. Am. Math. Soc. 205 (1975) 205-220.

[14] A.N. Kolmogorov, 'Three approaches to the quantitative definition of information', Probl. Inform. Transmission 1 (1965) 1-7.

[15] A.N. Kolmogorov V.A. Uspenskii, 'Algorithms and randomness', Theory Probab. Appl. 32 (1987) 389-412.

[16] P. Martin-Löf, The Definition of Random Sequences, Information and Control 9 (1966) 602619.

[17] R. Rado, 'Universal graphs and universal functions', Acta Arith. 9 (1964) 393-407.

[18] F.P Ramsey, 'On a problem of formal logic', Proc. London Math. Soc. 30 (1930) 264-286.

[19] E. Specker, 'Ramsey's Theorem does not hold in recursive set theory', Studies in logic and the foundations of mathematics (North-Holland, Amsterdam, 1971).

[20] P. Vitányi M. Li, An Introduction to Kolmogorov Complexity and Its Applications (SpringerVerlag, 1993). 